\title{
Faringitis aguda, ¿estreptocóccica? Empleo del estudio etiológico y de antimicrobianos en dos centros pediátricos universitarios
}

\author{
SEBASTIÁN MUÑOZ C., MARCELA CÓRDOVA A., \\ VIERA MORALES M. y LORENA CIFUENTES A.
}

\section{Sore throat, ¿streptococcal etiology? Use of etiological screening and antimicrobial prescription in two university pediatric centers}

The diagnosis and treatment of sore throat in pediatric patients attending one of two facilities at a university hospital: pediatric ambulatory facility (PAF) and emergency unit (EU) in a period of 14 months are described. There were 421 patients at the PAF and 289 at the EU, of whom $65 \%$ and $76 \%$ were studied for Streptococcus pyogenes respectively. Streptococcus were detected in both centers in $37 \%$ of cases, with a peak detection of $50 \%$ at ages 6 to 10 years. There were 217 patients without any bacteriological study, of whom $162(75 \%)$ received antibiotics. The importance of bacteriological confirmation prior to antibiotic prescription in this disease is emphasized.

Key words: Pharyngitis; Streptococcus pyogenes; Diagnosis; Management.

Palabras claves: Faringitis; Streptococcus pyogenes; Diagnóstico; Manejo.

\section{Introducción}

Las infecciones de la vía aérea superior constituyen la causa más frecuente de consulta en el grupo pediátrico ${ }^{1}$. Dentro de éstas, la faringoamigdalitis aguda (FA) ocupa un lugar importante. Streptococcus pyogenes es la causa bacteriana más común de esta patología, aislándose en 15 a $30 \%$ de los niños ${ }^{2}$ y en 5 a $10 \%$ de los adultos con $\mathrm{FA}^{3,4}$; el resto es principalmente de etiología viral $^{4,5}$. Se cree que otras bacterias de la microbiota orofaríngea pueden influir en el desarrollo de FA $^{6}$. Streptococcus pyogenes se puede aislar de secreción faríngea durante todo el año, siendo más frecuente en otoño y primavera ${ }^{7,8}$. Se utilizan dos métodos para su detección: tests inmunológicos ("rápidos") para la detección del antígeno grupo A de $S$. pyogenes en el carbohidrato $\mathrm{C}$ de su pared, y el cultivo bacteriológico de la secreción faríngea. Este último corresponde al estándar de oro por su alta sensibilidad y especificidad $^{9,10}$.

En muchos países se estima que la indicación de antimicrobianos en las consultas por $\mathrm{FA}^{11,12}$ varía desde 30 a $75 \%$; considerando que la gran mayoría corresponden a FA virales, se deduce que existiría una sobreindicación de ellos.

Por otro lado, la presunción clínica de infección estreptocóccica tiene, según diversos estu$\operatorname{dios}^{5-7}$, una sensibilidad de sólo 50 a $70 \%$ y especificidad de 60 a 80\%, en acertar la etiología. Debido al alto porcentaje de falsos positivos que representa emplear una presunción etiológica basada en manifestaciones clínicas, no se ha pro-

Pontificia Universidad Católica de Chile, Santiago, Chile:

Departamento de Pediatría. (LCA).

Residentes Servicio de Pediatría (SMC, MCA, VMM).

Estudio realizado en el Servicio de Pediatría Ambulatoria, Centro Médico San Joaquín y Servicio de Urgencia, Pontificia Universidad Católica de Chile.

Recibido: 13 abril 2004

Aceptado: 21 diciembre 2004 
ducido una reducción en el uso de antimicrobianos en la práctica clínica general ${ }^{12-15}$. Diversos estudios han evaluado la utilidad de síntomas y signos en identificar la infección estreptocóccica proponiendo el uso de scores clínicos para anticipar la etiología de las FA y desarrollando algoritmos de conducta, con el objetivo de disminuir el uso de antimicrobianos en la población estudiada ${ }^{16-18}$. Entre otros, el score de Centor ha sido el mejor validado en poblaciones adultas, con estudios prospectivos. McIsaac lo modificó para aplicarlo en población pediátrica, obteniendo su validación prospectiva, tanto en adultos como en niños. Este autor estratificó el riesgo de estreptococcia en cinco niveles, desde 1 a $51 \%$, con sensibilidad de $85 \%$ y especificidad de $92 \%{ }^{16,17}$. Sin embargo, cuando se ha intentado que médicos entrenados pongan en práctica estos algoritmos se ha fracasado en disminuir la indicación de antimicrobianos $^{19}$.

Recientemente se ha propuesto realizar tests inmunológicos para la detección rápida del antígeno A de $S$. pyogenes en pacientes que tengan un score clínico elevado y reservar la obtención de un cultivo bacteriológico para aquellos pacientes con score bajo, con lo que se podría disminuir los costos y el tiempo invertidos en el diagnóstico y tratamiento de la FA estreptocóccica ${ }^{20}$.

La optimización del diagnóstico etiológico en una FA aspira a minimizar las consecuencias de un diagnóstico incorrecto: sobreuso de antimicrobianos e incremento en la resistencia de los patógenos respiratorios en la comunidad (p. ej: Streptococcus pneumoniae, S. pyogenes), pérdida de tiempo, y el perjuicio al paciente al retardar un diagnóstico correcto (p. ej: adenovirosis, síndrome de mononucleosis infecciosa causado por VEB). Se han hecho esfuerzos en distintos países en generar guías de práctica clínica para el manejo de la FA y evitar así el uso excesivo de antimicrobianos ${ }^{21}$.

A pesar de contar actualmente con suficiente evidencia que apoya el uso de alguno de los métodos diagnósticos expuestos ${ }^{5,9-11,22-24}$, éstos aún no son usados universalmente en nuestro país, principalmente por no estar disponibles en todos los centros de atención ambulatoria de pacientes, por la demora en disponer del informe (en el caso del cultivo bacteriológico), y por su costo.

Los objetivos de este trabajo fueron hacer una breve caracterización clínica de las consultas por FA, describir la magnitud de la utilización de exámenes de laboratorio en FA (test inmunológico o cultivo) para el diagnóstico de $S$. pyogenes, cuantificar la indicación de antimicrobianos en pacientes con diagnóstico clínico de FA, y establecer la tasa de detección de este patógeno. Este estudio no pretende evaluar la utilidad de los signos clínicos en el diagnóstico de FA por $S$. pyogenes, sobre lo cual existe amplia literatura. (Al respecto recomendamos la lectura de las referencias $3,4,12,14,15-18,20,21)$.

\section{Pacientes y Métodos}

Se trabajó en forma retrospectiva con los registros clínicos de la Unidad de Morbilidad Aguda Pediátrica del Centro Médico San Joaquín de la Pontificia Universidad Católica de Chile (grupo A) y de la Unidad de Urgencia Pediátrica del Hospital Clínico de la misma universidad (grupo B). La revisión abarcó desde enero del 2002 a marzo del 2003 en el grupo A y desde enero del 2002 hasta diciembre del 2002 en el grupo B. Se seleccionaron aquellos registros que tenían el diagnóstico de faringitis, FA o amigdalitis, y a quiénes se les había solicitado un test inmunológico o cultivo bacteriológico para $S$. pyogenes*. En el grupo A se revisó la totalidad de registros clínicos del periodo, en el grupo B no se tuvo acceso a todo el periodo; por esta razón sólo se conoció la frecuencia de consulta por faringitis, amigdalitis y FA en el grupo A.

Se consignaron las siguientes características clínicas de los pacientes: edad, presencia de fiebre (temperatura axilar $\geq 38^{\circ} \mathrm{C}$ ), odinofagia, tos, dolor abdominal, vómitos, adenopatías submandibulares sensibles, halitosis, exudado faríngeo, petequias en el paladar blando y antecedente de contacto con un caso índice. La edad se obtuvo por la diferencia entre la fecha de consulta y la fecha de nacimiento. Los otros signos clínicos se consignaron como presentes sólo si estaban registrados así, asumiendo su ausencia si el médico no lo explicitara en su ficha. Debido a esto, probablemente obtuvimos un subregistro importante de la sintomatología/signología. A pesar que asumimos que un estudio restrospectivo no es adecuado para evaluar estas variables clínicas, lo realizamos considerando que el subregistro debería afectar por igual a todos los pacientes en un mismo centro.

Se empleó el score clínico descrito por $\mathrm{Mc}$ Isaac ${ }^{16,17}$ que contempla los siguientes parámetros:

\footnotetext{
* Aunque no se hubieran consignado los mencionados diagnósticos.
} 
- edad menor a 15 años, un punto (se aplicó a todos los pacientes), agregándose además un punto por cada uno de los siguientes signos presentes:

- fiebre $\left(>38^{\circ} \mathrm{C}\right)$

- ausencia de tos

- adenopatías submandibulares sensibles

- exudado faríngeo.

Se utilizó este score sólo para detectar si existían diferencias clínicas entre el grupo de pacientes a quienes que se les tomó exámenes y aquellos que no se estudiaron.

Se registró además la solicitud de exámenes para detectar $S$. pyogenes (test inmunológico o bacteriológico) y si se indicó antimicrobianos en esa consulta. Los resultados de los análisis inmunológicos y bacteriológicos se obtuvieron de los registros del laboratorio del hospital.

Los pacientes que no aparecieron en los registros del laboratorio (n: 217) fueron asignados al grupo sin exámenes, asumiendo que no les fueron solicitados.

Para evaluar cómo influye el tomar exámenes en la indicación de antimicrobianos, se incluyó a aquellos pacientes a los que se les tomaron ambos exámenes, en el grupo de test inmunológico. No se evaluaron conductas posteriores como la adherencia al tratamiento antimicrobiano o si se cambió la indicación inicial al conocer el resultado del cultivo.

Las muestras faríngeas se obtuvieron mediante hisopado con tórula de rayón para el test inmunológico y de algodón para el cultivo bacteriológico.

Test inmunológico. Se utilizó un test inmunocromatográfico (Test Pack Plus Strep $A\left({ }^{\circledR}\right)$ de Abbott, el cual tiene una sensibilidad de 90 a $95 \%$ según el fabricante.

Cultivo bacteriológico. Se realizó en agar sangre de cordero, incubación en atmósfera de $\mathrm{CO}_{2}(10 \%)$, efectuando la primera lectura a las 18-24 horas, como se encuentra estandarizado ${ }^{8}$. La certificación de la especie pyogenes se efectuó mediante un test de PYR positivo.

Análisis estadístico. Se utilizó el programa informático STATISTICA® (StatSoft) para la estadística descriptiva y para los análisis comparativos. Se utilizó el test de $\chi^{2}$ para comparar variables de dos valores posibles y el test ANOVA para ver las diferencias en variables categóricas de más de dos valores posibles como el puntaje clínico.

\section{Resultados}

En el período observado 710 registros clíni- cos cumplían con los criterios de inclusión. De ellos 421 provinieron del grupo A y 289 del grupo B. En el grupo A representaron el 4\% del total de consultas en ese período (n: 10.282). Los pacientes incluidos tenían un promedio de edad de 6 años 11 meses ( 6 meses - 19 años 10 meses); en 4 pacientes no se obtuvo la fecha de nacimiento. El paciente de menor edad en que se detectó S. pyogenes (cultivo faríngeo positivo) tenía 1 año 4 meses.

Los signos clínicos más registrados fueron: presencia de fiebre $\left(>38^{\circ} \mathrm{C}\right)$, ausencia de tos, adenopatías submandibulares sensibles, exudado faríngeo y odinofagia (Tabla 1).

En el grupo A se efectuó análisis de laboratorio para confirmar la etiología estreptocóccica a $65 \%$ de los pacientes, en el grupo B a $76 \%$. (Tabla 2). Es muy notorio cómo en el grupo B casi no se tomaron cultivos para detectar $S$. pyogenes y cómo la proporción de tests inmunológicos dentro del total es mucho más alta que en el grupo A. A 27 pacientes se les realizó tanto test inmunológico como cultivo bacteriológico (13 en el grupo A y 14 en el grupo B); los resultados de ambos exámenes fueron coincidentes en todos ellos, 4 fueron positivos y 23 negativos.

Se comparó el score clínico de McIsaac observado en los distintos grupos, en ambos centros mediante ANOVA. Los puntajes promedio fueron distintos entre ambos centros (ANOVA Kruskal-Wallis $\mathrm{p}=0,0008$ ), de manera que no eran poblaciones comparables. Sin embargo, dentro de cada centro no se observó relación entre el score clínico y la solicitud o no de uno de los análisis de laboratorio para detectar $S$. pyogenes.

La frecuencia de solicitud de exámenes e indicación de antimicrobianos no varió significativamente según la edad (Tabla 3). La mayor tasa de positividad se observó en una edad entre 6 y 10 años (50\%), disminuyendo conforme la edad se aleja de este rango.

La tasa global de detección de $S$. pyogenes fue de $37 \%$, (grupo A 33\%, grupo B 42\%) Al comparar mediante $\chi^{2}$ los grupos entre sí y entre las distintas técnicas, la tasa de detección fue similar en ambos centros e independiente del examen utilizado (datos no presentados).

De los pacientes en que no se realizaron exámenes para la detección de S. pyogenes, en ambos centros de atención pediátrica (n: 217), 162 $(74,7 \%)$ recibieron la indicación de antimicrobianos. Si se compara con la tasa de detección de S. pyogenes $(37 \%)$, la prescripción de antimicrobianos duplicó a la real indicación en estos centros $(\mathrm{p}<0,05)$. 
Tabla 1. Características clínicas de los pacientes con faringitis, frecuencia de detección de Streptococcus pyogenes e indicación de antimicrobianos

\begin{tabular}{|c|c|c|}
\hline & \multicolumn{2}{|c|}{ Total } \\
\hline & Positivo / total evaluado & Frecuencia $(\%)$ \\
\hline Temperatura $>38^{\circ} \mathrm{C}$ axilar & $623 / 710$ & 88 \\
\hline Ausencia de tos & $517 / 710$ & 73 \\
\hline Odinofagia & $439 / 710$ & 62 \\
\hline Exudado amigdalino & $375 / 710$ & 53 \\
\hline Adenopatías sensibles & $320 / 710$ & 45 \\
\hline Vómitos & $186 / 710$ & 26 \\
\hline Dolor abdominal & $150 / 710$ & 21 \\
\hline Petequias en paladar blando & $87 / 710$ & 12 \\
\hline Rash escarlatiniforme & $35 / 710$ & 5 \\
\hline Halitosis & $26 / 710$ & 4 \\
\hline Contacto con S. pyogenes & $11 / 710$ & 2 \\
\hline Puntaje McIsaac > 3 & $384 / 710$ & 54 \\
\hline S. pyogenes en hisopado faríngeo & $182 / 493 * *$ & 37 \\
\hline Indicación de AM* & $375 / 710$ & 53 \\
\hline
\end{tabular}

* AM: antimicrobianos.

** El denominador es menor en el rubro detección de $S$. pyogenes, ya que no se tomó exámenes a todos los pacientes.

Tabla 2. Score clínico de McIsaac, según examen utilizado para la detección de Streptococcus pyogenes en dos centros pediátricos

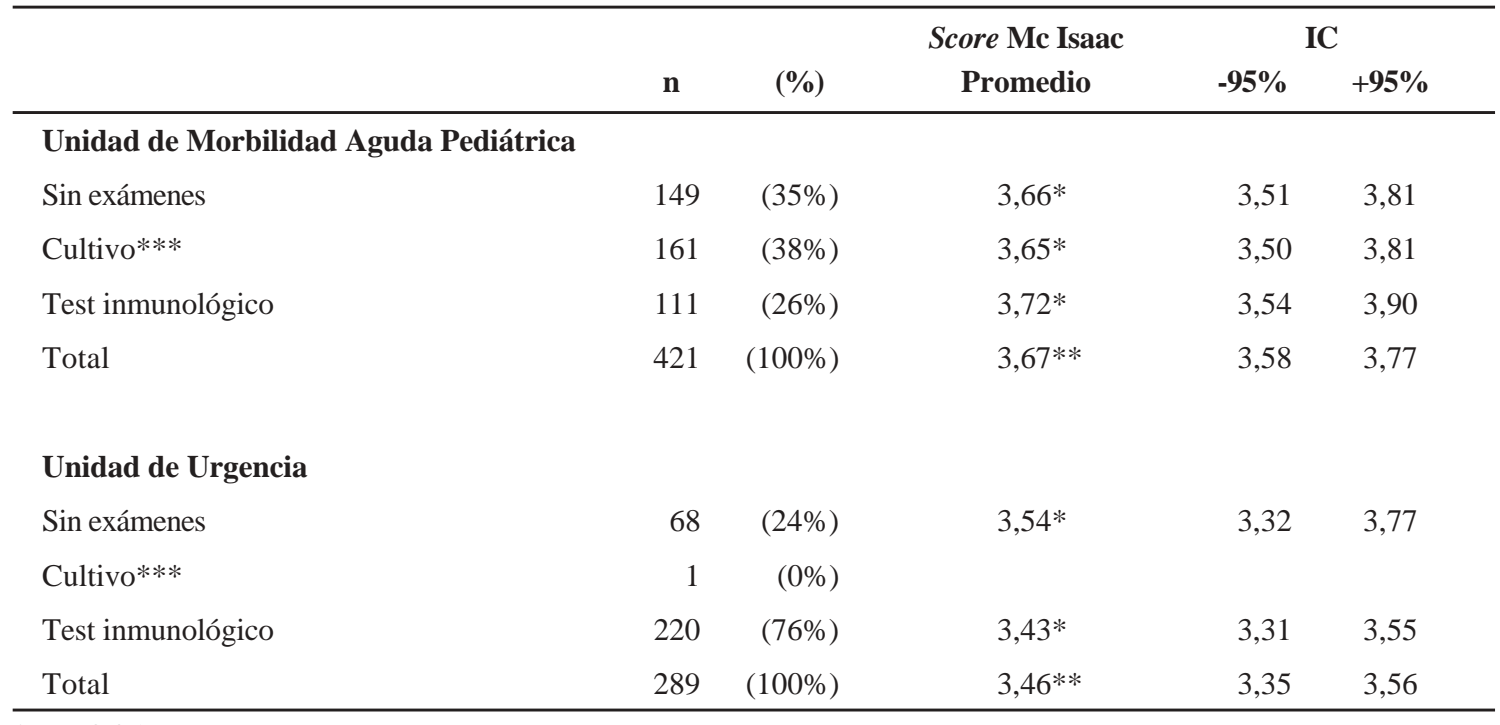

$* \mathrm{p}>0,05$

$* * \mathrm{p}=0,0008$ (ANOVA Kruskal-Wallis)

***Corresponden a aquellos cultivos obtenidos sin test inmunológico en paralelo. Los pacientes a los que se les tomó ambos tests, se incluyeron sólo en el grupo de test inmunológico (ver Material y Métodos). 
Tabla 3. Prescripción de antimicrobianos y detección de Streptococcus pyogenes según edad

\begin{tabular}{lrccc}
\hline $\begin{array}{c}\text { Rango de edad } \\
\text { (años) }\end{array}$ & $\mathbf{n}$ & $\begin{array}{c}\text { Indicación de } \\
\mathbf{A M}(\%)\end{array}$ & $\begin{array}{c}\text { Con exámenes de } \\
\text { laboratorio }(\mathbf{n} / \%)\end{array}$ & $\begin{array}{c}\text { S. pyogenes } \\
\text { positivos }(\%)\end{array}$ \\
\hline 0 a 2 & 21 & 19,1 & $16 /(76,2)$ & 6,3 \\
> 2 a 4 & 113 & 40,7 & $29 /(72,6)$ & 23,2 \\
> 4 a 6 & 205 & 53,7 & $145 /(70,7)$ & 33,8 \\
> 6 a 8 & 146 & 58,9 & $101 /(69,2)$ & 49,5 \\
> 8 a 10 & 77 & 50,7 & $53 /(68,8)$ & 50,9 \\
> 10 a 12 & 72 & 63,9 & $45 /(62,5)$ & 37,8 \\
> 12 a 14 & 51 & 58,8 & $36 /(70,6)$ & 41,7 \\
> 14 a 16 & 18 & 55,6 & $9 /(50,0)$ & 33,3 \\
Mayor a 16 & 3 & 33,3 & $2 /(66,7)$ & 0,0 \\
Total & 706 & 52,7 & $489 /(69,3)$ & 37,0 \\
\hline
\end{tabular}

\section{Discusión}

La frecuencia de consultas por infecciones respiratorias agudas (IRAs) en la Unidad de Morbilidad Aguda de nuestra institución, en un periodo de 3 años, asciende en promedio a $66 \%$ de las consultas totales; dentro de ellas el predominio categórico de las IRAs altas (78\%, comunicación personal L. Cifuentes) coincide plenamente con lo descrito en otros estudios nacionales ${ }^{1}$. El diagnóstico de FA se consignó en 4\%, lo que corresponde a $8 \%$ de las IRAs altas. No disponemos de datos provenientes de otros centros pediátricos nacionales, ya que casi no existe información al respecto. Considerando el gran número de patologías que están incluidas en el diagnóstico de IRA alta y que además el diagnóstico de FA varía con la edad, podemos afirmar que es un porcentaje importante dentro de nuestras consultas.

La observación de las características clínicas de los pacientes con FA fue imperfecta por tratarse de un estudio retrospectivo. No obstante, las características clínicas de los pacientes que consultaron por FA fueron comparables a las descritas en otros estudios ${ }^{11,17,24}$. Nuestro análisis demuestra que el sesgo del diseño afectó por igual a los pacientes con y sin análisis de laboratorio; sin embargo, este sesgo fue distinto en cantidad de morbilidad aguda que en la Unidad de Urgencia, observándose en esta última, scores clínicos más bajos. Se podría pensar que los pacientes acuden a un servicio de emergencia con mayor precocidad en el desarrollo de su enfermedad o, tal vez, que en la Unidad de Urgencia los pediatras están sujetos a mayor presión asistencial en ciertos momentos. Por otro lado, en la Unidad de Morbilidad Aguda del centro ambulatorio, prácticamente toda la atención médica es ligada a actividades docentes de pre y postgrado, no así en la Unidad de Urgencia, lo cual podría implicar una mayor rigurosidad en los registros médicos en la primera. Para conocer mejor la correlación de los signos clínicos con presencia de infección por $S$. pyogenes se requieren estudios prospectivos $\mathrm{y}$ controlados. Existen pocos estudios en pacientes pediátricos, y hasta el momento, se ha descrito que el score clínico tiene una sensibilidad que no supera al $60 \%$ para la detección de enfermedad por S. pyogenes ${ }^{20}$.

En nuestra experiencia hubo total concordancia entre los resultados de los dos exámenes para detectar S. pyogenes; sin embargo, sólo a 27 pacientes se les realizó ambos tests. No existen en nuestro centro evaluaciones de la sensibilidad y especificidad de los tests inmunológicos versus cultivo bacteriológico. La Academia Americana de Pediatría (2002) no recomienda la realización de un cultivo bacteriológico para confirmar los casos que resultaren negativos con el test inmunológico, siempre y cuando se haya demostrado que, en el medio donde trabaja el médico, el test inmunológico tiene una sensibilidad similar al cultivo bacteriológico ${ }^{24}$. Sin embargo, hay quienes estiman aceptable utilizar un segundo test inmunológico como confirmatorio ${ }^{10}$. Algunos autores proponen realizar detección rápida de antígeno en pacientes que tengan un score clínico alto y reservar el cultivo bacteriológico para aquellos con score clínico bajo, con lo que se podría 
disminuir costo y tiempo en el diagnóstico y tratamiento de una FA estreptocóccica; estiman además innecesario confirmar la negatividad de un test inmunológico mediante cultivo bacteriológico, ya que el valor predictor negativo del primero sería suficiente para la toma de decisiones ${ }^{20}$.

Este estudio revela que en nuestra realidad la sobreindicación de antimicrobianos es muy semejante a lo descrito en otros lugares, y aunque se dispone de métodos de diagnóstico, éstos sólo se usan en algunas ocasiones. No encontramos diferencias clínicas que explicaran porqué en ciertas circunstancias se solicitaron estos exámenes y en otras no. Es obvio que las razones para solicitar exámenes no son solamente clínicas, dependen también de las circunstancias de la consulta. Por ejemplo, en la Unidad de Urgencia se solicitaron 220 tests inmunológicos y 15 cultivos bacteriológicos, en cambio en la Unidad de Morbilidad Aguda se realizaron 111 tests inmunológicos y 174 cultivos bacteriológicos. Influyen en la toma de decisiones por el médico el costo de los exámenes y la disponibilidad de seguros de salud por el paciente, el grado de confianza que sienta el médico respecto a que el paciente rescatará el resultado del examen bacteriológico y acudirá a control. Sin embargo, si consideramos que este estudio está realizado en un centro universitario donde la actividad es mayoritariamente docente-asistencial, se podría esperar una aún mayor sobreindicación de antimicrobianos en centros médicos no académicos de nuestro país.

Diversos centros han evaluado el uso de scores clínicos para la correcta indicación de antimicrobianos en FA, ya que esta patología, junto con otitis media aguda, dan cuenta de los principales diagnósticos con que se indican antimicrobianos en la práctica pediátrica ambulatoria. El uso de estos scores ha sido recomendado como una alternativa aceptable, pero los hallazgos en pediatría han sido algo distintos, de manera que parecen ser poco útiles ${ }^{17,18,20}$. Por otro lado, la implementación de tales scores clínicos no ha logrado resolver el problema de la excesiva prescripción de antimicrobianos ${ }^{19}$.

El presente estudio permite apreciar en forma categórica que, si el médico clínico solicita algún análisis de laboratorio para confirmar la presencia de $S$. pyogenes, podrá reducir hasta en $50 \%$ el número de tratamientos antimicrobianos que indique en FA, en comparación con lo que se indica en casos en que sólo se guía por hallazgos clínicos. Esto apoya fuertemente la recomendación del uso de estos exámenes para lograr mayor certeza diagnóstica.
Es necesario optimizar el proceso diagnóstico de una FA por las consecuencias que derivan de un diagnóstico incorrecto. El sobrediagnóstico implica someter a un paciente de manera innecesaria a un tratamiento antimicrobiano durante 7 a 10 días con el consiguiente riesgo de reacciones de hipersensibilidad y otros efectos adversos, además del costo económico para esa familia y la contribución al aumento de resistencia bacteriana en la población. Por otro lado, no diagnosticar o no tratar una FA estreptocóccica encierra potenciales complicaciones a largo plazo ${ }^{21}$.

Concordamos, en base a la información disponible hasta ahora, con los autores que proponen que sólo se debieran indicar antimicrobianos en FA con confirmación bacteriológica ${ }^{20,24}$, y que ésta se debiera solicitar cada vez que se dude de la etiología de una FA.

Actualmente estamos llevando a cabo un estudio prospectivo que intenta responder cuál es la correlación entre los hallazgos clínicos en FA y la presencia de $S$. pyogenes.

\section{Resumen}

Se describen aspectos clínicos, del diagnóstico y tratamiento de faringitis en pacientes pediátricos, 421 en un centro ambulatorio y 289 en la unidad de emergencia de un hospital universitario, que consultaron en un periodo de 14 meses. En los centros respectivos hubo 65 y $76 \%$ de pacientes en que se realizaron exámenes para documentar la etiología estreptocóccica. Se demostró la existencia de Streptococcus pyogenes en $37 \%$ de los explorados, siendo similar en ambos centros, con una incidencia máxima de 50\% en el grupo etario de 6 a 10 años. En 217 pacientes no se realizó estudio etiológico, de éstos $162(75 \%)$ recibieron tratamiento antimicrobiano. Se enfatiza la importancia de los exámenes de confirmación bacteriológica para la correcta indicación de antimicrobianos en esta patología.

\section{Agradecimientos}

A Tamara Hirsch B. por su estímulo y por la revisión crítica del manuscrito, a Hernán Cofré por el apoyo estadístico.

\section{Bibliografía}

1.- López I M, Sepúlveda H, Valdés I. Acute respiratory illnesses in the first 18 months of life. Rev Panam Salud Pública 1997; 1: 9-17.

2.- Kaplan E L, Top F H Jr, Dudding B A, Wannamaker L W. Diagnosis of streptococcal pharyngitis: 
differentiation of active infection from the carrier state in the symptomatic child. J Infect Dis 1971; 123: 490-501.

3.- Komaroff A L, Pass T M, Aronson M D, Ervin C T, Cretin S, Winickoff $\mathrm{R} \mathrm{N}$, et al. The prediction of streptococcal pharyngitis in adults. J Gen Intern Med 1986; 1: 1-7.

4.- Poses R M, Cebul R D, Collins M, Fager S S. The accuracy of experienced physicians' probability estimates for patients with sore throats: implications for decision making. JAMA 1985; 254: 925-9.

5.- Hamrick H J, Mangum M E. Beta-hemolytic Streptococcus milleri group misidentified as Streptococcus pyogenes on throat culture. Pediatr Infect Dis J 1999; 18: 75-6.

6.- Mihajlovic-Ukropina M, Roncevic N. Significance of normal oropharyngeal flora in the development of streptococcal pharyngitis and outcome of penicillin therapy. Med Preg1 1998; 51: 275-8.

7.- Del Pont J M. Capítulo 196. Faringoamigdalitis. En Meneghello J, Fanta E, Paris E, Puga T. Pediatría. Quinta Edición. 1997. Editorial Médica Panamericana S.A. Buenos Aires, Argentina. pp: 1226-8.

8.- Ruoff K, Whiley R, Beighton D. Streptococcus. En: Murray P, Baron E, Pfaller M, Tenover F, Yolken R. Manual of Clinical Microbiology. $8^{\text {th }}$ edition, 2003. American Society for Microbiology. Washington University School of Medicine, Washington, USA, p 405-21.

9.- Sociedad Latinoamericana de Infectología Pediátrica. Consenso sobre el diagnóstico y tratamiento de la faringoamigdalitis estreptocóccica. Rev Chil Infect 1999; 16: 218-9.

10.- Gieseker K E, Roe M H, MacKenzie T, Todd J K. Evaluating the American Academy of Pediatrics diagnostic standard for Streptococcus pyogenes pharyngitis: backup culture versus repeated rapid antigen testing. Pediatrics 2003; 111 (6 Pt 1): e666-70.

11.- Kaplan E L, Amren D P. Diagnosing streptococcal pharyngitis. JAMA 1992; 268: 599-600.

12.- Dobbs F. A scoring system for predicting group A streptococcal throat infection. Br J Gen Pract 1996; 46: 461-4.

13.- Wolter J M. Management of a sore throat. Antibiotics are no longer appropriate. Aust Fam Physician 1998; 27: $279-81$.
14.- Steinhoff M C, Abd el Khalek M K, Khallaf N, Hamza H S, el Ayadi A, Orabi A, et al. Effectiveness of clinical guidelines for the presumptive treatment of streptococcal pharyngitis in Egyptian children. Lancet 1997; 350: 918-21.

15.- McGinn T G, Deluca J, Ahlawat S K, Mobo B H Jr, Wisnivesky J P. Validation and modification of streptococcal pharyngitis clinical prediction rules. Mayo Clin Proc 2003; 78: 289-93. Erratum in: Mayo Clin Proc 2003; 78: 1313

16.- McIsaac W, White D, Tannenbaum D, Low D E. A clinical score to reduce unnecessary antibiotic use in patients with sore throat. Can Med Assoc J 1998; 158: 75-83.

17.- McIsaac W, Goel V, To T, Low D. The validity of a sore throat score in family practice. Can Med Assoc J 2000; 163: 811-5.

18.- Wald E R, Green M D, Schwartz B, Barbadora K. A streptococcal score card revisited. Pediatr Emerg Care 1998, 14: 109-11.

19.- McIsaac W J, Goel V, To T, Permaul J A, Low D E. Effect on antibiotic prescribing of repeated clinical prompts to use a sore throat score: lessons from a failed community intervention study. J Fam Pract 2002; 51: 339-44.

20.- Cohen R, Levy C, Ovetchkine P, Boucherat M, WeilOlivier C, Gaudelus J, de la Rocque F, et al. Evaluation of streptococcal clinical scores, rapid antigen detection test and cultures for childhood pharyngitis. Eur J Pediatr 2004; 163: 281-2.

21.- Del Mar C B. A clinical prediction model did well in diagnosing pediatric group A beta-hemolytic streptococcal pharyngitis. ACP J Club 2002; 136: 37.

22.- Mayes T, Pichichero M E. Are follow-up throat cultures necessary when rapid antigen detection tests are negative for group A streptococci? Clin Pediatr (Phila) 2001; 40: 191-5.

23.- Schroeder B M. Diagnosis and management of group A streptococcal pharyngitis. Am Fam Physician 2003; 67: $880,883-4$

24.- Bisno A L, Gerber M A, Gwaltney J M Jr, Kaplan E L, Schwartz R H. Infectious Diseases Society of America. Practice guidelines for the diagnosis and management of group A streptococcal pharyngitis. Infectious Diseases Society of America. Clin Infect Dis 2002; 35: 113-25.

Correspondencia a:

Sebastián Muñoz Concha

smunoz@puc.cl 


\section{Sociedad Chilena de Infectología}

\section{Actividades año 2005}

\section{CURSOMICROBIOLOGÍA CLÍNICA}

"Visión del médico clínico sobre requerimientos del laboratorio clínico"

Organiza: Comité Microbiología Clínica

Fecha: Lunes 9 y Martes 10 de mayo (medio día) 2005

Sede: Santiago - Hotel Torre Mayor

Objetivo: Actualizar aspectos relevantes del diagnóstico microbiológico, estudios de susceptibilidad antimicrobiana, análisis de calidad y relación del Laboratorio de Microbiología con otros servicios de apoyo como banco de sangre.

Directora: Olivia Trucco A.

Coordinadora: Béatrice Hervé E.

\section{CURSO: "LAS INFECCIONES INTRAHOSPITALARIAS EN EL MUNDO PEDIÁTRICO”}

Organiza: Comité Infecciones Intrahospitalarias

Fecha: Junio

Sede: Santiago

Objetivo: Capacitar al equipo de salud en estrategias en prevención y control de infecciones nosocomiales.

Director: Luis Delpiano M.

Coordinador: Jaime Labarca L.

\section{CURSO VACUNAS 2005}

Co-organizado por Sociedad Chilena de Infectología-Universidad del Desarrollo-Universidad de Chile y Pontificia Universidad Católica de Chile.

Fecha: Agosto

Sede: Santiago

Objetivos: - Lograr conocimientos actualizados sobre las vacunas existentes y nuevas vacunas

- Comprender los criterios de priorización para la incorporación de nuevas vacunas en el PAI dirigidas a niños, adolescentes y adultos

- Reconocer los criterios considerados en los cambios en el PAI vigente

- Conocer modelos de vigilancia de eventos adversos a vacuna

- Comprender los desafíos de la Influenza y las estrategias de enfrentamiento de una posible pandemia.

Directora: M. Teresa Valenzuela B.

\section{CURSO DE TERAPIA ANTIRETROVIRAL}

Fecha: Agosto 2005

Sede: Santiago

Objetivo: Actualización de diagnóstico y tratamiento en infección por VIH

Directora: Ana Chávez P.

Coordinadora: Teresa Bidart $\mathrm{H}$.

\section{CURSOITINERANTE ENREGIONES}

Fecha: a definir

Sedes: Antofagasta, Coquimbo y Castro.

Objetivo: Permanencia de 2 días de infectólogo de adultos y pediatra, más un microbiólogo y enfermera de control de infecciones, para dictar curso teórico y práctico en las áreas definidas de común acuerdo

Directora: Tamara Hirsch B.

\section{CONGRESO CHILENO DE INFECTOLOGÍA}

Fecha: 26 al 29 de octubre de 2005

Sede: Puerto Varas

Presidenta Comité Científico: María Elena Santolaya de P. 